\title{
Article \\ Species-Specific Duplication and Adaptive Evolution of a Candidate Sex Pheromone Receptor Gene in Weather Loach
}

\author{
Lei Zhong ${ }^{1,2} \mathbb{D}$, Weimin Wang ${ }^{2}$ and Xiaojuan Cao ${ }^{2, *(\mathbb{D}}$ \\ 1 Fisheries Research Institute, Wuhan Academy of Agricultural Sciences, Wuhan 430207, China; \\ leiclock@163.com \\ 2 College of Fisheries, Key Lab of Agricultural Animal Genetics, Breeding and Reproduction of Ministry of \\ Education/Key Lab of Freshwater Animal Breeding of Ministry of Agriculture and Rural Affairs, \\ Huazhong Agricultural University, Wuhan 430070, China; wangwm@mail.hzau.edu.cn \\ * Correspondence: caoxiaojuan@mail.hzau.edu.cn
}

Citation: Zhong, L.; Wang, W.; Cao, X. Species-Specific Duplication and Adaptive Evolution of a Candidate Sex Pheromone Receptor Gene in Weather Loach. Genes 2021, 12, 1845. https://doi.org/10.3390/genes 12121845

Academic Editors: Nico M Van Straalen and Lukáš Kratochvíl

Received: 15 June 2021

Accepted: 19 November 2021

Published: 23 November 2021

Publisher's Note: MDPI stays neutral with regard to jurisdictional claims in published maps and institutional affiliations.

Copyright: (c) 2021 by the authors. Licensee MDPI, Basel, Switzerland. This article is an open access article distributed under the terms and conditions of the Creative Commons Attribution (CC BY) license (https:// creativecommons.org/licenses/by/ $4.0 /)$.

\begin{abstract}
The release and sensation of sex pheromone play a role in the reproductive success of vertebrates including fish. Previous studies have shown that the weather loach Misgurnus anguillicaudatus perceives sex pheromones by olfaction to stimulate courtship behavior. It was speculated that weather loaches use smell to recognize intraspecific mates. However, the identification of loach pheromone receptor has not been reported. By comparative transcriptomic approach, we found that the olfactory receptor gene or114-1 was male-biasedly expressed in the olfactory epithelium of M. anguillicaudatus, M. bipartitus and the closely related species Paramisgurnus dabryanus. This sex-biased expression pattern implicated that or114-1 presumably encoded a sex pheromone receptor in loaches. M. bipartitus and P. dabryanus, like zebrafish, possess one or114-1 only. However, in M. anguillicaudatus, or 114-1 has two members: Ma_or114-1a and Ma_or114-1b. Ma_or114-1a, not Ma_or114-1b, showed sex-differential expression in olfactory epithelium. Ma_or114-1b has base insertions that delayed the stop codon, causing the protein sequence length to be extended by 8 amino acids. Ma_or114-1a was subject to positive selection resulting in adaptive amino acid substitutions, which indicated that its ligand binding specificity has probably changed. This adaptive evolution might be driven by the combined effects of sexual selection and reinforcement of premating isolation between the sympatric loach species.
\end{abstract}

Keywords: pheromone receptor; olfaction; gene duplication; loach; differential expression; adaptive evolution

\section{Introduction}

The sensory system and its signal source play an important role in the pre-mating reproductive isolation between species [1,2]. Similar to the visual system and its signals, the chemical sensory system (including smell and taste) and chemical signals also participate in the evolution of genetic communication barriers [1,3]. Pheromone is a chemical signal, a molecule or a fixed combination of molecules, released by the organism to stimulate the instinctive response of other individuals within the same species, and it is widely present in various groups in the animal kingdom [4]. Pheromone is sensed by smell mainly by binding to olfactory receptors [5,6]. As a type of pheromone, sex pheromone has the function of attracting the opposite sex in the same species, promoting reproductive synchronization and inducing reproductive behavior $[7,8]$. In the past 10 years, sex pheromones have been identified in many species, including fish, with their extremely diverse chemical properties. For example, they are mostly long-chain hydrocarbons in insects and include steroids and peptides in vertebrates $[9,10]$. Correspondingly, receptors of sex pheromones have evolved very differently in different animal groups [7].

The sex pheromone-olfactory system is highly species-specified [4]. Pheromone signals can achieve specificity in two ways: using a single unique molecule or a specific combination of multiple molecules [7]. If the genes related to the synthesis of sex pheromone 
mutate, it may lead to the production of new molecular structures or new combinations of multiple molecules, which will lead to changes in their specificity. On the other hand, variations in the amino acid sequence of sex pheromone receptors will cause changes in the binding specificity of sex pheromone molecules [7]. When the sex pheromone to be sensed is a combination of multiple molecules, what is needed is a combination of multiple sex pheromone receptors [11]. Therefore, the mutation of a single sex pheromone receptor can also bring new specificity of perceiving sex pheromones at the combinatorial level.

In fish, the sex pheromone-olfactory system is related to the successful conduction of the reproduction process, which has been confirmed by the research on the sex pheromoneolfactory system of goldfish and others [12-14]. The identified sex pheromones in fish mainly include sex steroids, prostaglandins and bile acids [12]. The identification of fish sex pheromone receptors lags behind that of mammals, while the sex pheromone receptors identified in vertebrates are from olfactory receptors [10]. Olfactory receptors in vertebrates mainly include OR (odorant receptor), TAAR (trace amine-associated receptor), V1R (vomeronasal type 1 receptor) and V2R (vomeronasal type 2 receptor) families, belonging to the 7-transmembrane $\mathrm{G}$ protein-coupled receptor super family, with ligand binding sites in the transmembrane domain $[15,16]$. There are more than 300 members of these four families in zebrafish [17]. OR, TAAR, V1R, and V2R families all have members that assume the function of pheromone receptors in vertebrates $[10,18]$. Among them, the role of OR114-1 and V1R2 (ORA1) as sex pheromone receptors has been functionally verified in zebrafish [18-20].

Through whole-genome sequencing and olfactory epithelial transcriptome sequencing, olfactory receptors can be efficiently identified. In addition to whole-genome sequencing, fishes of which the olfactory epithelial transcriptomes have been sequenced include: Danio rerio [21,22], Carassius auratus [23], Senegalese sole [24], Anguilla anguilla [17], Oncorhynchus keta [25], Coilia nasus [26], Megalobrama amblycephala [27], which suggests that transcriptome sequencing is a feasible method for olfactory receptor identification.

Weather loaches (fishes in the genus Misgurnus and Paramisgurnus), which are of important aquacultural and medicinal values in East Asia, have a well-developed sense of smell due to their benthic living habits and accompanying visual degradation. Studies have shown that weather loaches perceive sex pheromones by olfaction to stimulate courtship behavior [28,29], and it is speculated that they use smell for species-specific mate recognition. Therefore, weather loaches have the potential to be models for the study of sex pheromone and its perception system. However, the direct identification and functional verification of loach pheromone receptors have not been previously reported.

Males have a perception different from that of females to the same sex pheromone. To arouse different perception of one ligand in different sexes, there are mainly two ways: the expression of the receptors is different in different sexes or, the higher brain circuitry is sexually dimorphic. The former is more direct. Therefore, although a sex pheromone receptor is not necessarily sex-differentially expressed, we could expect that a part of sex pheromone receptors might have a sex-differential expression pattern. On the other hand, if an olfactory receptor has a sex-differential expressed pattern in olfactory epithelium, it is highly probable that the olfactory receptor plays a role of sex pheromone receptor.

In this study, by comparative transcriptomic approach, we tried to find candidate sex pheromone receptors in the weather loaches through the identification of male/female differentially expressed olfactory receptors, and to investigate their molecular evolution.

\section{Materials and Methods}

\subsection{Sample Collection}

The sample collection and following experiments were conducted in accordance with the national legislation of China and approved by the Ethics Committee of Huazhong Agricultural University. We collected adult samples of M. anguillicaudatus, M. bipartitus and P. dabryanus in Yueyang, Zhoushan, Daqing and Honghu of China (Table 1). The taxonomic classification of the samples relied on the morphological characteristics given by Chen [30]. 
The sex identification of weather loach was based on the morphological sexual dimorphism: the males have a small bony plate on each pectoral fin that the females don't have [31]. Because there are natural diploid and tetraploid populations in Misgurnus anguillicaudatus [32,33], we employed a flow cytometry (CyFlow Space, Sysmex Partec, Germany) to detect the DNA content of erythrocytes to determine the ploidy status of each sample. The peripheral blood of each loach was collected from the tail vein using a syringe. Then the blood cells were suspended and stained in $1 \mathrm{~mL}$ staining buffer (Cystain DNA 1 step staining solution, Sysmex Partec, Germany) for $1 \mathrm{~min}$. Erythrocytes of Paramisgurnus dabryanus have a relative DNA content of diploid and their corresponding fluorescence intensity was used as control. The diploid individuals were leaved for subsequent experiments.

Table 1. Sample information.

\begin{tabular}{|c|c|c|c|}
\hline Species & Sample Number & Sex & Location \\
\hline \multirow{10}{*}{ Misgurnus anguillicaudatus } & B1 & Female & Yueyang \\
\hline & B2 & Male & Yueyang \\
\hline & YYAB1 & Female & Yueyang \\
\hline & YYAB2 & Male & Yueyang \\
\hline & YYM2 & Male & Yueyang \\
\hline & YYM3 & Male & Yueyang \\
\hline & YYM4 & Male & Yueyang \\
\hline & YYM6 & Female & Yueyang \\
\hline & YYM8 & Female & Yueyang \\
\hline & ZS2 & Female & Zhoushan \\
\hline \multirow{6}{*}{ Misgurnus bipartitus } & $\mathrm{C} 1$ & Female & Daqing \\
\hline & $\mathrm{C} 2$ & Male & Daqing \\
\hline & DQB52 & Female & Daqing \\
\hline & DQB53 & Female & Daqing \\
\hline & DQB54 & Male & Daqing \\
\hline & DQB56 & Male & Daqing \\
\hline \multirow{8}{*}{ Paramisgurnus dabryanus } & D1 & Female & Honghu \\
\hline & D5 & Male & Honghu \\
\hline & D7 & Female & Honghu \\
\hline & YYD1 & Male & Yueyang \\
\hline & YYD2 & Male & Yueyang \\
\hline & YYD3 & Male & Yueyang \\
\hline & YYD5 & Female & Yueyang \\
\hline & YYD6 & Female & Yueyang \\
\hline
\end{tabular}

\subsection{Transcriptome Sequencing and Analyses}

\subsubsection{Tissue Sampling and RNA Extraction}

One pair of male and female mature individuals from each species (M. anguillicaudatus (B1, B2), M. bipartitus (C1, C2) and P. dabryanus (D1, D5)) was sampled during the mating period. After anesthesia, the olfactory epithelium of each individual was dissected under a microscope and then respectively used for total RNA extraction. The total RNA was extracted using TRIzol reagent (Invitrogen, USA). The gDNA eraser (TaKaRa, China) was used to remove the genomic DNA in the process of RNA isolation. 


\subsubsection{Library Construction and Sequencing}

Library preparation and sequencing were carried out by BGI (Shenzhen, China). Briefly, after mRNA isolation of the total RNA, libraries for transcriptome sequencing were constructed using the NEBNext Ultra RNA Library Prep Kit for Illumina. Agilent 2100 Bioanaylzer and ABI StepOnePlus Real-Time PCR System were employed in the qualification and quantification of the libraries. One sequencing library was constructed for each individual. Therefore, there are a total of 6 libraries. Using the Illumina HiSeq 4000 platform (Illumina, San Diego, CA, USA), at least 5Gb data (150 bp paired-end reads) was produced for each library. The derived reads were deposited into the Sequence Read Archive of the National Center for Biotechnology Information (NCBI) (Accession No. SRR14800568-14800573).

\subsubsection{Bioinformatic Analyses}

Sequence Assembly and Annotation

After filtering the data of the next-generation sequencing, the Trinity program was employed for De novo assembly [34], and then the Tgicl program was adopted to remove the redundancy to obtain non-redundant transcript sequences [35]. The reads of the three species $M$. anguillicaudatus, $M$. bipartitus and $P$. dabryanus were assembled separately. In this way, the transcript sequence sets of each of the three species could be obtained. The functions of the transcripts were annotated using NT, NR, SwissProt, COG, KEGG and GO databases. The olfactory receptor genes were extracted according to the functional annotation.

\section{DEG Detection}

First, the Bowtie 2 program was employed to calculate the expression level of the 6 samples, and then the PossionDis method [36] was adopted to detect differentially expressed genes (DEG) between male and female samples of the same species. The intersection of olfactory receptor genes and differentially expressed genes were inferred as candidate sex pheromone receptor genes for subsequent analyses.

\section{Ortholog Identification}

The CDSs of transcripts were predicted based on the alignments of the functional annotation. Then BLAST and phylogenetic analyses were adopted to determine the orthologous relationships of the sequences. According to the candidate sex pheromone receptor gene sets and the orthology, the orthologous candidate sex pheromone receptor genes of the three species could be obtained. The phylogenetic analyses were performed by the maximum likelihood method (ML) under the general time-reversible (GTR) model in the MEGA7 software [37]. The statistical confidence of each node was assessed with 500 bootstrap replicates. The phylogenetic analyses were also performed by the Bayesian method under the GTR model in the MrBayes 3.1.2 program [38]. Two independent Bayesian analyses were run simultaneously for 10 million generations each sampling every 100th generation. A burn-in of 25,000 trees was removed. The statistical confidence in the nodes of the Bayesian tree was assessed by posterior probabilities.

\subsection{Genomic PCR, Sequencing and Related Analyses}

Genomic DNA was extracted by a high-salt method from the fin rays of each individual loach preserved previously in ethanol (a part of samples in Table 1) and then used as a polymerase chain reaction (PCR) template. Based on the 5'UTR and 3'UTR sequences of the transcripts (Supplementary Materials Additional file 1), primers conserved among the three species were designed. The or114-1 genes were amplified by PCR for each of the species. For high fidelity, PrimeSTAR Max DNA polymerase (TaKaRa, Dalian, China) was adopted in the amplification reaction.

The reaction system was $50 \mu \mathrm{L}$, including 10 pmol per primer, 200 ng genomic DNA and $25 \mu \mathrm{L}$ the PrimeSTAR Max premix. 
PCR program: $95^{\circ} \mathrm{C}$ for $5 \mathrm{~min}$, followed by 30 consecutive cycles of the following process: $94{ }^{\circ} \mathrm{C}$ for $45 \mathrm{~s}, 55^{\circ} \mathrm{C}$ for $45 \mathrm{~s}$ and $72{ }^{\circ} \mathrm{C}$ for $1 \mathrm{~min}$.

Primer sequences:

Forward: GGAGAGAGATTTTGTAGATGCTGC;

Reverse: GACAATCACACACAAAAGAGAATG

Each PCR product was purified and sequenced. When double peaks or multiple peaks appeared on the sequencing peak map, the purified PCR product was cloned using the Mighty TA-cloning Reagent Set for PrimeSTAR®kit (TaKaRa, Dalian, China) and E. coli DH5 $\alpha$ Competent Cells (TaKaRa, Dalian, China). At least 6 positive clones were sequenced from each individual of $M$. bipartitus and P. dabryanus on an ABI 3730 capillary sequencer (Applied Biosystems, Foster City, USA). At least 20 positive clones were sequenced for each individual of $M$. anguillicaudatus to ensure that each allele has two monoclonal representatives due to $M$. anguillicaudatus having two or114-1 paralogs inferred from the transcriptomic data. The derived representative gene sequences were deposited to GenBank (Accession No. MZ065164-MZ065167; for allele sequences, see Supplementary Materials Additional file 2).

The phylogenetic analyses of the obtained sequences were conducted by the ML method under the GTR model in the MEGA7 software. The statistical confidence of each node was assessed with 500 bootstrap replicates. The phylogenetic analyses were also performed by the Bayesian method under the GTR model in the MrBayes 3.1.2 program. Two independent Bayesian analyses were run simultaneously for 10 million generations each sampling every 100th generation. A burn-in of 25,000 trees was removed. The statistical confidence in the nodes of the Bayesian tree was assessed by posterior probabilities.

\subsection{Quantitative RT-PCR Assays}

First, three pairs of mature male and female individuals from each of the three species were obtained as biological replicates, and the olfactory epithelium of each individual was sampled (Misgurnus anguillicaudatus, female: B1, YYM6, YYM8, male: YYM2, YYM3, YYM4; M. bipartitus, female: C1, DQB52, DQB53; male: C2, DQB54, DQB56; Paramisgurnus dabryanus, female: D7, YYD5, YYD6; male: YYD1, YYD2, YYD3). Total RNA was extracted from each sample.

Then, the RevertAid First Strand cDNA Synthesis Kit (Thermo Scientific, Vilnius, Lithuania) was used to reverse transcribe mRNA into cDNA.

Next, The Bio-Rad CFX Connect Real-Time PCR Detection System was employed for quantitative RT-PCR (qRT-PCR) analysis. The reagent used was the Power SYBR Green PCR Master Mix (Applied Biosystems). The analyses were carried out separately for each of the three species. Three technical replicates were prepared for each sample. $\beta$-actin was used as the internal reference gene and its primers were designed based on conservative sequences of the three species. Primers that specifically amplify Ma_or114-1 $a$ and Ma_or114-1b were designed based on the unique sequences, and were used in qRT-PCR after verification by PCR sequencing. According to the transcript sequences, the primers were designed to amplify Mb_or114-1 (M. bipartitus) and Pd_or114-1 (P. dabryanus) in qRT-PCR. The primer sequences used are shown in Table 2.

The PCR program is described in Table 1. A melting curve analysis was performed to ensure unique amplification for each primer pair. The relative expression levels of male and female samples of a certain species were quantified by the comparative $C_{T}\left(2^{-\Delta \Delta C T}\right)$ method [39].

Different individuals of the same species are divided into two groups according to males and females (three biological replicates in each group). Based on the relative quantification of the expression of the same gene in samples of the same species, a twosample $\mathrm{F}$ test was used to evaluate the homogeneity of variance, and then a two-sample $t$ test was conducted to examine whether the gene expression level of male loach was significantly up-regulated relative to that of female loach. 
Table 2. Primers for qRT-PCR.

\begin{tabular}{|c|c|c|c|}
\hline Primer Name & Primer Sequence $\left(5^{\prime}-3^{\prime}\right)$ & $\mathrm{TM}\left({ }^{\circ} \mathrm{C}\right)$ & Product Length $(\mathrm{bp})$ \\
\hline$\beta$-actin $\mathrm{F}$ & GGGTATGGAGTCTTGCGGTA & 58.88 & 131 \\
\hline$\beta$-actin $\mathrm{R}$ & CAGCAATGCCAGGGTACATG & 59.26 & \\
\hline Ma_or114-1a F & AAGATCTACGTGTGATATGTACC & 59.08 & 140 \\
\hline Ma_or114-1a R & TTAATGCAGATTAACCTTATTAGAAG & 58.20 & \\
\hline Ma_or114-1b F & GAGATCTACGTGTGCTATGTAC & 58.89 & 150 \\
\hline Ma_or114-1b R & GGGAAGACCACTGATGTAGAT & 58.78 & \\
\hline$M b \_o r 114-1 \mathrm{~F}$ & GCCATCATTTTCCССTTGCA & 59.10 & 119 \\
\hline Mb_or114-1 R & CCCAGCAAACCAACCATTGA & 58.95 & \\
\hline Pd_or114-1 F & TGTGTTTGTGAACACACATCA & 58.00 & 163 \\
\hline Pd_or114-1 R & CGCCTGAAGGAGAACGAAAT & 59.72 & \\
\hline
\end{tabular}

The PCR program used in the experiment is: (1) $95.0^{\circ} \mathrm{C}$ for $3: 00 \mathrm{~min}(2) 95.0^{\circ} \mathrm{C}$ for $0: 10 \mathrm{~min}(3) 55.0{ }^{\circ} \mathrm{C}$ for $0: 20 \mathrm{~min}(4) 72.0{ }^{\circ} \mathrm{C}$ for $0: 20 \mathrm{~min}$ (5) $75.0^{\circ} \mathrm{C}$ for 0:05 min (+ Plate Read) (6) GOTO 2, 40 more times (7) Melt Curve 65.0 to $95.0^{\circ} \mathrm{C}$, increment $0.5{ }^{\circ} \mathrm{C} / \mathrm{s}(0: 05 \mathrm{~min}+\mathrm{Plate}$ Read).

\subsection{Analysis of Adaptive Evolution}

First, based on the or114-1 sequences of the three loach species, we tested whether the evolution rate of the target branch of the gene tree was significantly different to and faster than that of other branches, that is, whether the target branch's $\omega(\omega=\mathrm{dN} / \mathrm{dS}$, the ratio of the non-synonymous substitution rate to the synonymous substitution rate) was different to and significantly greater than that of other branches. The codeML program in the PAML package was adopted to perform statistical tests based on the branch model [40]. The input tree is ((Ma_or114-1a,Ma_or114-1b),Mb_or114-1),Pd_or114-1). Likelihood values were calculated under one-ratio model and two-ratio model. Under the two-ratio model, the target branch was set as the foreground branch and other branches as the background branch, and the respective $\omega$ of the foreground branch and the background branch were calculated. The likelihood ratio test was first performed on the likelihood values under the two-ratio model and the one-ratio model to infer whether the $\omega$ of the target branch was significantly different to and significantly greater than that of other branches. Next, the likelihood ratio test was performed on the likelihood values calculated under the two-ratio model and the two-ratio model (limited $\omega 1=1$ ) to infer whether the $\omega$ of the target branch is significantly greater than 1.

Because positive selection may only occur at a few sites of a specific branch instead of all sites, the next step was to use the codeML program to perform a positive selection test based on the branch-site model [40]. The target branch was set as the foreground branch, and the other branches were set as the background branch. The likelihood ratio test was performed on the likelihood values under Model A and Model A (limited $\omega 2=1)$. At the same time, the BEB method attached to Model A can calculate the posterior probabilities of different types of sites and identify sites subject to positive selection.

The online program RELAX was adopted to test whether the change in the evolution rate of the target branch relative to the reference branch was due to the relaxation of purifying selection or the increase of positive selection [41]. aBSREL [42] and BUSTED [43], which are alternative selection testing methods based on the branch-site model, were also used to run tests for adaptive evolution, and both tests can be performed online. The website of RELAX, aBSREL, BUSTED online programs is: http:/ / www.datamonkey.org (accessed on 8 April 2018).

Transmembrane regions of Ma_OR114-1 was predicted by the TMpred server (https: / / embnet.vital-it.ch/software/TMPRED_form.html accessed on 25 March 2018) [44].

\section{Results and Discussion}

\subsection{Identification of a Male-Biasedly Expressed or Gene}

In the sex-differentially expressed gene sets preliminarily identified by the bioinformatic analysis on the transcriptomic data of olfactory epithelium, one olfactory receptor gene was found showing male-biased expression in all the three loach species 
(M. anguillicaudatus, M. bipartitus and P. dabryanus) (Supplementary Materials Additional file 1, Table S1), hence it was presumably a candidate gene encoding sex pheromone receptor. Intriguingly, after checking the gene annotations, we found that this gene was orthologous to zebrafish or114-1 which has been functionally verified as a sex pheromone receptor [18]. Zebrafish or114-1 belongs to the A subfamily of OR family [45]. In zebrafish, the male mating behavior is stimulated by or114-1 mediated perception of prostaglandin F2 $\alpha$ and its derivatives [18]. Since or114-1 likely expressed sex-differentially in the olfactory epithelium of the three loach species which belong to the order Cypriniformes but in a basal branch different from zebrafish, this observation together with the previous study raised the possibility that or114-1 acts as a sex pheromone receptor at least in Cypriniformes.

\subsection{Species-Specific Duplication of or $114-1$}

In the searching for the or114-1 sequences in the assembled transcripts, we found that in M. anguillicaudatus, there were two distinct or114-1 haplotypes (named Ma_or114-1a and Ma_or114-1b) with considerable differences in sequence (more than 40 pairwise differences in 936 positions in the alignment), while M. bipartitus and P. dabryanus, and also D. rerio, each have only one or114-1. The CDSs of or114-1a of M. anguillicaudatus and or114-1 of M. bipartitus, $P$. dabryanus and D. rerio are all 942 bp (314 aa) in length. Ma_or114-1b has insertions within the 3'end of the CDS, resulting in the stop codon being shifted and the CDS length being extended to 966bp (322aa). Ma_or114-1a showed male-biased expression in M. anguillicaudatus as or114-1 did in M. bipartitus and P. dabryanus, whereas there was no significant difference between male and female expression of Ma_or114-1b (Table S1). Since there is only one or114-1 in zebrafish, and also in M. bipartitus and P. dabryanus, it could be inferred that the two or114-1 in $M$. anguillicaudatus were paralogs produced by lineagespecific gene duplication. Phylogenetic tree reconstruction was performed using the ML method based on the alignment of or114-1 sequences of the three loach species and zebrafish. In the resulting gene tree, the two or114-1 of $M$. anguillicaudatus first clustered with each other, then together clustered with or114-1 of M. bipartitus (Figure 1). This tree topology suggests that the gene duplication event which produced Ma_or114-1a and Ma_or114-1b in the genome of M. anguillicaudatus occurred in the branch leading to M. anguillicaudatus after the divergence of the ancestors of $M$. anguillicaudatus and M. bipartitus, that is, this gene duplication is species-specific to M. anguillicaudatus.

In order to determine whether the two or114-1 (Ma_or114-1a and Ma_or114-1b) of the weather loach are indeed paralogs rather than alleles, we surveyed the or114-1 sequences of more individuals. If $M a \_o r 114-1 a$ and $M a \_o r 114-1 b$ are paralogs in the weather loach genome, then it is expected that Ma_or114-1a and Ma_or114-1b exist in every individual of the loach, and possibly each of the two paralogs has two different alleles in some individuals. If Ma_or114-1 $a$ and Ma_or114-1b are actually two alleles in one locus instead, then only one of the two or114-1 types would appear in some individuals because this locus has a probability to be homozygous. We conducted genomic PCR using conservative primers and subsequent cloning and sequencing to obtain the or114-1 haplotype sequences of several loach individuals (three of $M$. anguillicaudatus, two of $M$. bipartitus, and two of $P$. dabryanus), and then underwent phylogenetic analysis (using zebrafish or114-1 as an outgroup). The result showed that different individuals of $M$. anguillicaudatus all possessed the two or114-1 types which were initially identified as paralogs; Ma_or114-1a and Ma_or114-1b each have 1 to 2 alleles in each individual; individuals of $M$. bipartitus and $P$. dabryanus, the same as zebrafish, each only possessed one or114-1 sequence or two or114-1 sequences which were slightly different from each other (less than 10 pairwise differences in 936 positions in the alignment) and thus more likely alleles rather than paralogs (Figure 2; Supplementary Materials Additional file 2). Therefore, it was proven that Ma_or114-1a and Ma_or114-1b of M. anguillicaudatus are not alleles, but true paralogs. 


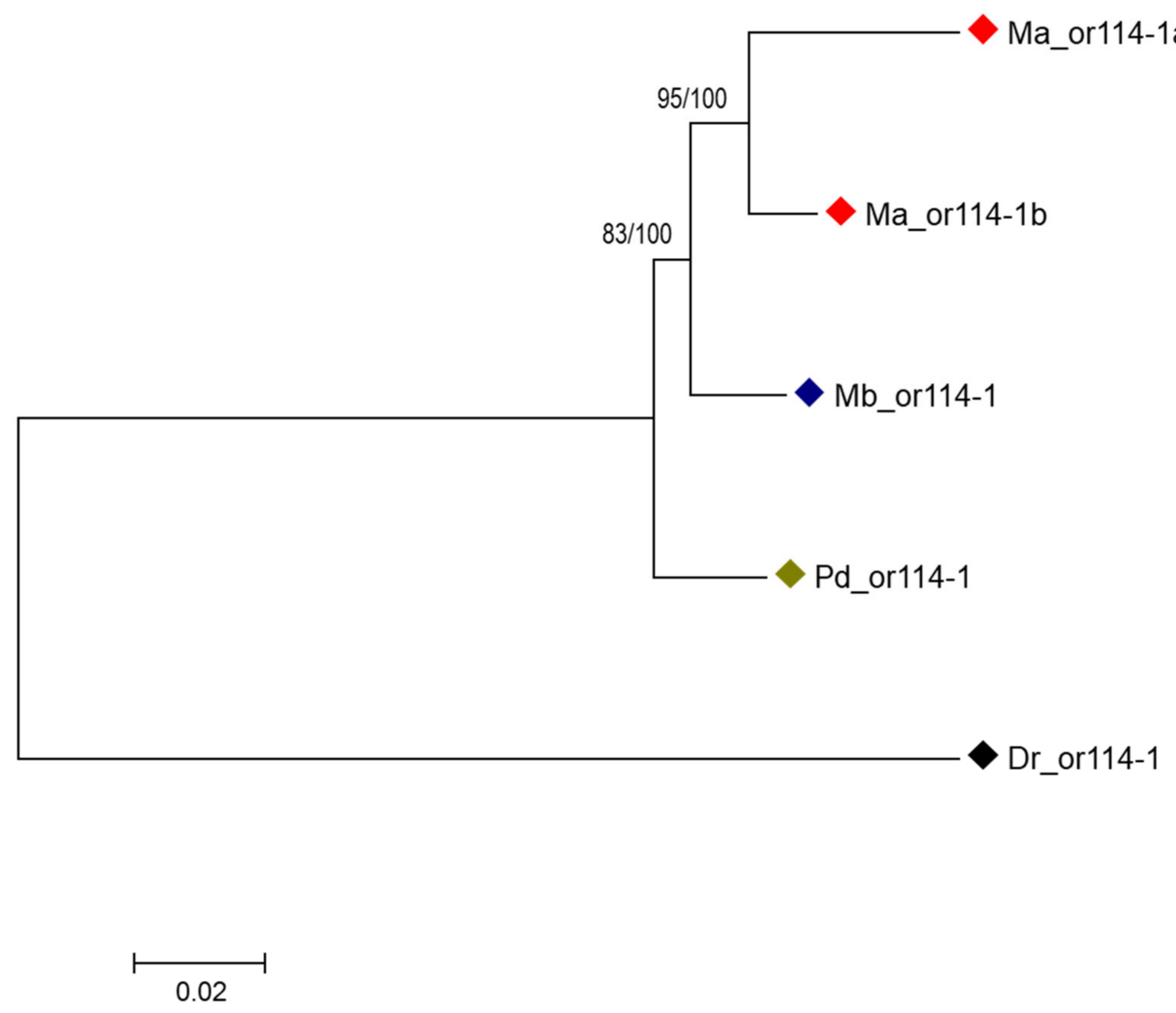

Figure 1. Phylogenetic tree of or114-1 of the three loach species and zebrafish. ML tree is shown and the general topologies of Bayesian tree is as the same as that of ML tree. Two types of statistical support values for nodes are provided: the former number is the percentage of support calculated with 500 bootstrap replicates in the ML tree reconstruction and the second number is posterior probabilities in the Bayesian reconstruction. Ma, Misgurnus anguillicaudatus; Mb, M. bipartitus; Pd, Paramisgurnus dabryanus; Dr, Danio rerio. GenBank Accession number of Dr_or114-1: NM_001128582.

Additionally, taking advantage of the ongoing loach genome sequencing project of the weather loach, we performed BLAST searches with the assembled sequences obtained by genome sequencing and found that Ma_or114-1a and Ma_or114-1b were in the same chromosome but not in the same contig. We compared the $5 \mathrm{~kb}$ flanking sequences both upstream and downstream of or114-1 in the two contigs and found there to be no obvious similarity between the flanking regions of the contigs (Supplementary Materials Additional file 3). This result indicated that the two or 114-1 were located at different loci of the loach genome, thus further supporting that $M a \_$or 114-1 $a$ and $M a \_$or 114-1b are paralogs produced by gene duplication. Meanwhile, whether the duplication of or114-1 in the loach took place by tandem duplication, segmental duplication or retrotransposition is still an open question. 


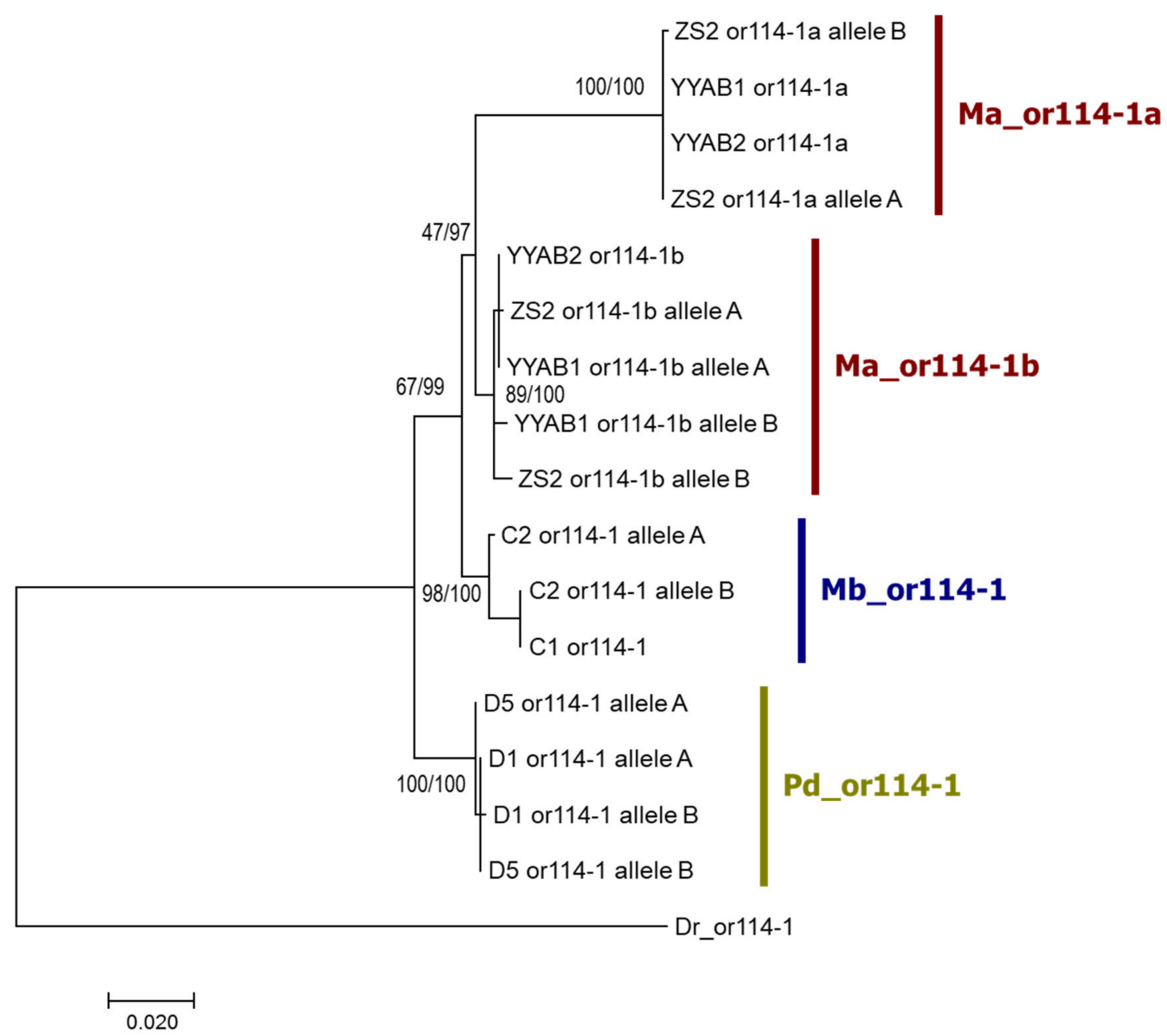

Figure 2. Phylogenetic tree of or114-1 alleles of the three loach species. or114-1 of zebrafish was used as an outgroup. ML tree is shown and the general topologies of Bayesian tree is as the same as that of ML tree. Two types of statistical support values for nodes are provided: the former number is the percentage of support calculated with 500 bootstrap replicates in the ML tree reconstruction and the second number is the posterior probabilities in the Bayesian reconstruction. Abbreviations of species names are as in Figure 1. Individual numbers are as in Table 1.

\section{3. qRT-PCR Verification of the Sex-Differential Expression}

We added biological replicates for each species to perform quantitative expression analyses of or114-1 by qRT-PCR to verify the preliminary conclusion of male-biased expression drawn from the bioinformatic analysis on RNA-seq data. Based on the qRT-PCR derived data, statistical tests were conducted to identify the significance of the expression difference between males and females. To the expression of Ma_or114-1a, the homogeneity of variance of the male group and the female group was not refused according to the result of F-test $(\mathrm{F}=0.04824, P=0.09203>0.05)$, and the $t$-test under the assumption of variance homogeneity produced a significant result $(t=-2.41623, P=0.03653<0.05)$, which indicated that the expression of $M a \_o r 114-1 a$ in males was significantly higher than that in females (Figure 3). The same method was used to analyze the expression of Ma_or114-1b, and the result showed that there was no significant difference between males and females (Figure 3), suggesting that Ma_or114-1b might have undergone neo- or subfunctionalization and no longer assumed the previous role of sex pheromone receptor. The same analysis was also performed on the expression of or114-1 in M. bipartitus and P. dabryanus. The results showed that in these two species the expression levels in males were all higher than those in females of the same species despite the difference not being statistically supported $(P>0.05, t$-test) (Figures S1 and S2). The insignificant $t$-test results were possibly caused by 
that the expression levels of or114-1 in olfactory epithelium differed greatly between male individuals, which resulted in large variance, and the number of biological replicates was relatively limited.

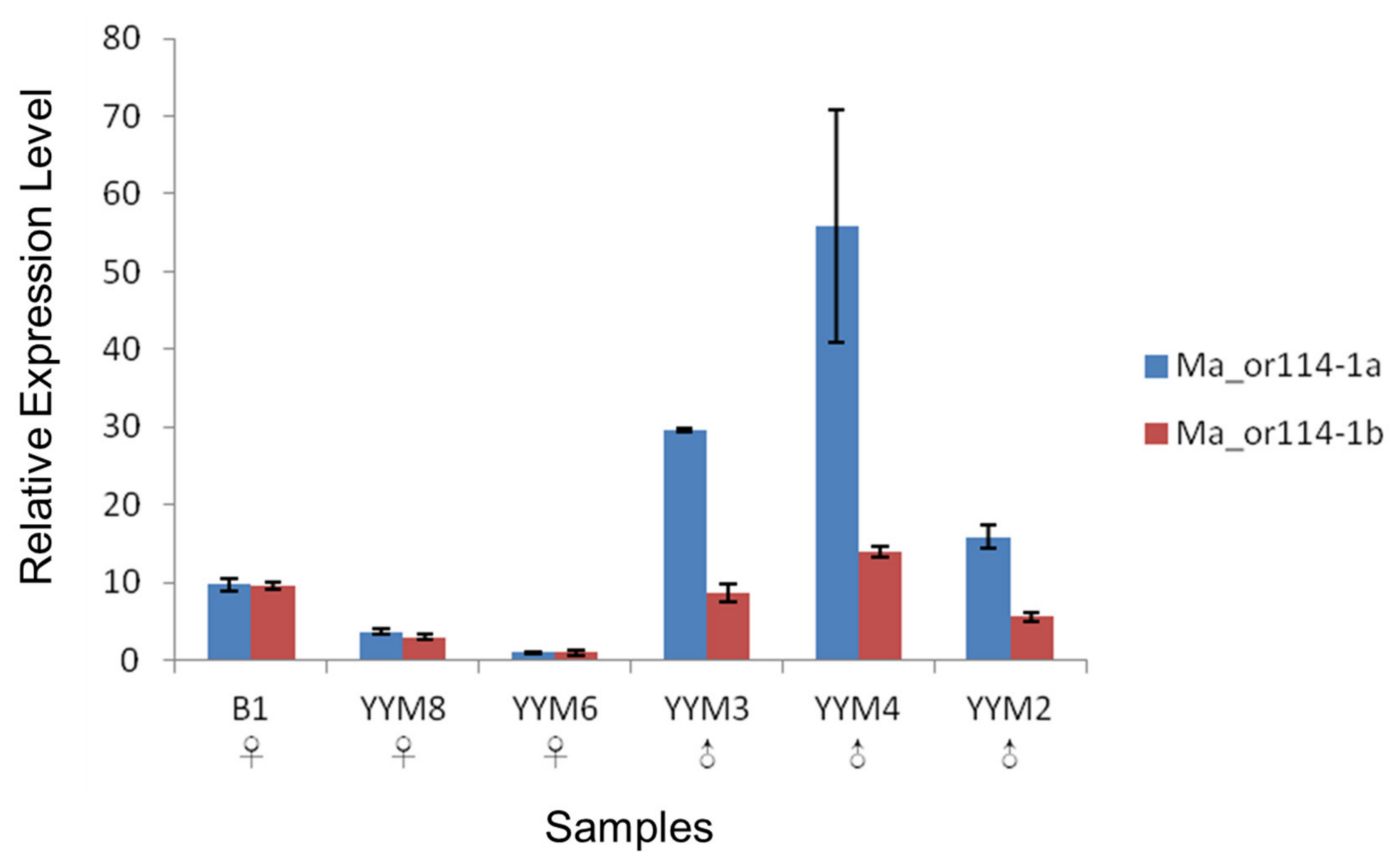

Figure 3. The relative expression levels of Ma_or114-1a and Ma_or114-1b in different male and female individuals of Misgurnus anguillicaudautus, based on the relative quantitative results of qRT-PCR $\left(2^{-\Delta \Delta C T}\right)$. Three technical replicates for each individual and each gene, and the error bar is the standard deviation of the three technical replicates. The sample numbers are as shown in Table 1 . When comparing expression difference of Ma_or114-1a between the male group and the female group, $\mathrm{F}=0.04824, P=0.09203>0.05 ; t=-2.41623, P=0.03653<0.05$ (equal variance assumed); significant. When comparing expression difference of $M a \_$or $114-1 b$ between the male group and the female group, $\mathrm{F}=1.123, P=0.94206>0.05$; $t=-1.37163, P=0.12104>0.05$ (equal variance assumed), not significant.

\subsection{Adaptive Evolution of or114-1 in Weather Loach}

In the reconstructed gene tree of or114-1, including orthologs of M. anguillicaudatus, M. bipartitus, P. dabryanus and D. rerio (as the outgroup), the or114-1s of M. anguillicaudatus, namely Ma_or114-1a and Ma_or114-1b, clustered together (Figure 1), suggesting that they were products of a gene duplication event that occurred in M. anguillicaudatus after the divergence of $M$. anguillicaudatus and M. bipartitus. Meanwhile, the branch of Ma_or114-1a was significantly longer than that of $M a \_$or114-1b, and also longer than the branches of or114-1 of $M$. bipartitus and P. dabryanus (Figure 1), suggesting that the evolution rate of Ma_or114-1a has accelerated. This acceleration of evolution rate could be caused by either positive selection or relaxed purifying selection. In order to test the prediction of adaptive evolution of Ma_or114-1a under positive selection, we conducted a series of tests based on the or114-1 sequences of $M$. anguillicaudatus, $M$. bipartitus and P. dabryanus using multiple tools.

We first employed the codeML program to perform the statistical test based on the branch model [40]. In the first step, we test whether the evolution rate of the branch Ma_or114-1a was significantly faster than that of other branches, that is, whether the $\omega$ of the branch $M a \_o r 114-1 a$ was significantly larger than that of other branches. Under the two-ratio model, the $\omega$ (1.461) of the Ma_or114-1a branch set as the foreground branch was larger than the $\omega(0.136)$ of the other branches as the background branch. The likelihood ratio test was performed on the likelihood values under the two-ratio model and the one-ratio model, and the result was significant $(P<0.001)$ (Table 3$)$, indicating that the evolution rate of $M a \_o r 114-1 a$ branch was significantly faster than other branches. In the 
next step, we test whether the branch $M a$ or $114-1 a$ was subject to positive selection under the branch model, that is, whether the $\omega$ of this branch was significantly greater than 1 . The likelihood ratio test was performed on the likelihood values under the two-ratio model and the two-ratio model (limited $\omega 1=1)$, and the statistical result was not significant $(P>0.05)$ (Table 3). Therefore, the test based on the branch model was ineffective to tell whether the branch of Ma_or114-1a was subject to positive selection or relaxed selective constraints.

Table 3. Test result under branch model by codeML.

\begin{tabular}{|c|c|c|c|}
\hline Model & $\log \mathrm{L}$ & Parameters & LRT \\
\hline M0 (one-ratio) & -1717.957 & $\omega=0.376$ & M0 vs. two-ratio: \\
\hline two-ratio model & -1708.171 & $\begin{aligned} \text { wbackground } & =0.136, \\
\omega \text { foreground } & =1.461\end{aligned}$ & $\begin{array}{c}2 \triangle \mathrm{\iota}=19.57, \mathrm{df}=1 \\
p<0.001\end{array}$ \\
\hline two-ratio model $(\omega 1=1)$ & -1708.506 & $\begin{aligned} \text { wbackground } & =0.137 \\
\omega \text { foreground } & =1.000\end{aligned}$ & $\begin{array}{c}\text { two-ratio }(\omega 1=1) \text { vs. two-ratio: } \\
\qquad \begin{array}{c}\mathrm{L} \Delta=0.67, \mathrm{df}=1, \\
p>0.05\end{array}\end{array}$ \\
\hline
\end{tabular}

The RELAX test can be used to determine whether the acceleration of evolution rate of the target branch relative to the reference branch for a certain gene tree is due to the relaxation of purifying selection pressure or the increase of positive selection pressure [41]. Based on the or114-1 sequences of $M$. anguillicaudatus, M. bipartitus and P. dabryanus, we set Ma_or114-1 $a$ as the target branch and performed RELAX test. The result of the test for selection intensification was significant $(\mathrm{K}=12.30, p<0.001)$ (Table S2), indicating that the selection pressure on the Ma_or114-1a branch has increased rather than relaxed, namely, the accelerated evolution of $\mathrm{Ma} \_$or114-1 $a$ was caused by positive selection rather than relaxed purifying selection.

The branching model is not highly effective in testing positive selection due to the fact that positive selection may only occur at a few sites rather than all sites in a gene. Next, we performed a positive selection test based on the branch-site model in the codeML program [40]. The Ma_or114-1a branch was set as the foreground branch, and the other branches were set as the background branch. The likelihood ratio test was performed on the likelihood values under Model A and Model A (limited $\omega 2=1$ ), and the statistical result was significant $(P<0.05)$ (Table 4$)$. This result supported that $M a \_$or114-1a has been positively selected at some sites after diverging from Ma_or114-1b. At the same time, as the BEB method attached to Model A can be used to calculate the posterior probability of different site types, 28 sites subject to positive selection were identified (Table 4). Compared with the predicted transmembrane domain protein model based on the amino acid sequence, it was found that positive selection sites were distributed in the 7 transmembrane domains, 3 extramembrane loops, the third inner loop and the N-terminal out of the membrane (Figure 4). Among them, the TM3, TM5, and TM6 transmembrane domains, which combine to form a pocket structure, have 1, 5, and 3 positively selected amino acid sites, respectively. Previous studies have confirmed that point mutations on TM3, TM5, and TM6 can change the ligand binding specificity of olfactory receptors [46]. The amino acid substitutions in these three domains on Ma_or114-1a were expected to change the binding profile of ligands. These amino acid substitutions that led to changes in ligand binding specificity were subject to positive selection, which means that these amino acid substitutions brought adaptive evolution.

At the same time, we performed aBSREL and BUSTED tests based on the branchsite model to further verify whether $M a$ or $114-1$ was subject to positive selection. Both tests yielded statistically significant results $(P<0.05$, Tables S3 and S4), which further supported that at least one amino acid site of Ma_or114-1a has been positively selected during evolution. 
Table 4. Test result under branch-site model by codeML.

\begin{tabular}{cccc}
\hline Model & $\log \mathrm{L}$ & LRT & Sites under Positive Selection \\
\hline Model A & -1705.590 & $2 \triangle \mathrm{l}=4.04$, & $6 \mathrm{~L}, 20 \mathrm{~S} * *, 41 \mathrm{~S}, 50 \mathrm{~V}, 74 \mathrm{Q}, 90 \mathrm{~K}, 105 \mathrm{M}, 136 \mathrm{~F}, 138 \mathrm{~T}, 148 \mathrm{~F}, 158 \mathrm{~T}, 164 \mathrm{I} *, 188$ \\
Model A $(\omega 2=1)$ & -1707.570 & $\mathrm{df}=1$, & $\mathrm{I}, 193 \mathrm{R}, 208 \mathrm{~T}, 215 \mathrm{~F}, 219 \mathrm{~V}, 225 \mathrm{M}, 226 \mathrm{~S}, 234 \mathrm{~W} * 2$, 235 H, 247 A, 248 M, 249 F \\
& & $p<0.05$ & $*, 265 \mathrm{~S}, 269 \mathrm{~K}, 274 \mathrm{I}, 281 \mathrm{~V}$ \\
\hline
\end{tabular}

NOTE. The amino acid sites with the ${ }^{* *}, *$ or no superscript denote the sites with the posterior probability $<0.01, P<0.05$ and $P<0.1$ respectively.

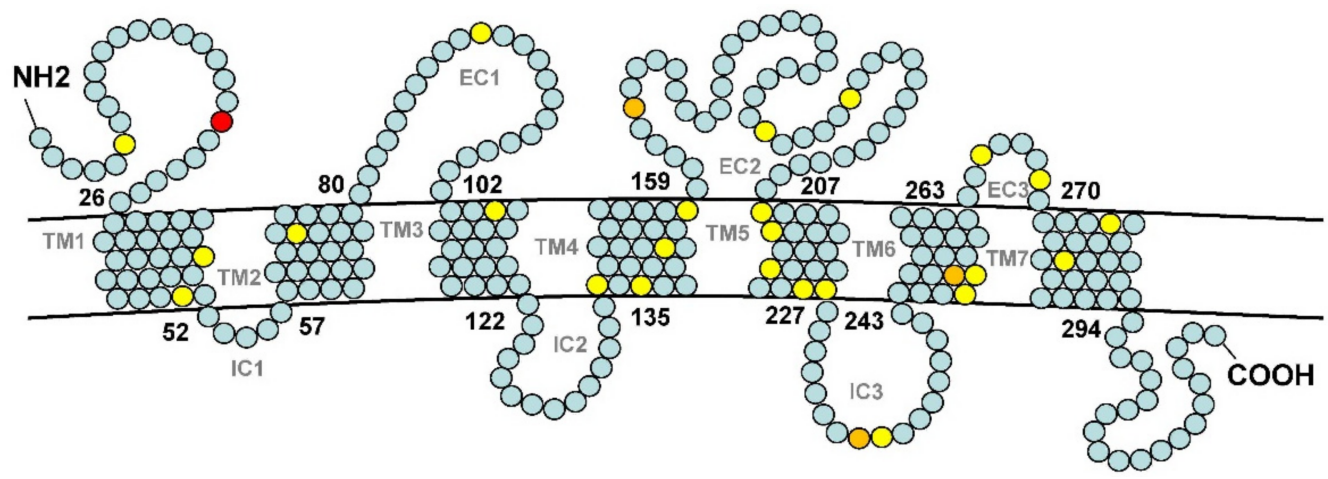

Figure 4. The predicted protein structure of Ma_OR114-1a of M. anguillicaudatus. The detected amino acid sites subject to positive selection are indicated in red (posterior probability $<0.01)$, orange $(P<0.05)$ and yellow $(P<0.1)$. It is worth noting that point mutations in the transmembrane domains TM3, TM5, and TM6 can change the ligand binding specificity of the olfactory receptor. IC: inside the membrane; EC: outside the membrane.

Genes related to reproduction often have a faster evolutionary rate due to sexual selection [3]. If as inferred, OR114-1 has the function of sex pheromone receptor and is closely related to mate recognition, one plausible explanation for the force of adaptive evolution of Ma_or114-1a would be sexual selection. The ancestral OR114-1 might be pluripotency and have other basic functions besides the sex pheromone receptor function (e.g., functions for perceiving the odors of food); hence it expressed both in males and females, and might be subject to strict evolutionary constraints in $M$. anguillicaudatus before the gene duplication and in the related species. According to the evolutionary model of gene duplication [47], after the duplication of or114-1 in M. anguillicaudatus, the two copies then might undergo possible functional differentiation. Ma_or 114-1b probably inherited some other basic functions (subfuntionalization), while Ma_or114-1a more specifically assumed the role of sex pheromone receptor. As a result, the evolutionary constraints imposed on Ma_or114-1a were relaxed, namely purifying selection is weakened, while the driving force of sexual selection would be prominent, hence the amino acid sequence was subject to positive selection and adaptive amino acid substitution occurred.

Since $M$. anguillicaudatus and P. dabryanus live in the same habitats and the breeding seasons overlap, there might be a process of reinforcement between the two species [48-50]. This process strengthens the pre-mating reproductive isolation through the differentiation of mating-related traits of the two species [51,52]. Due to the similar morphology and benthic life of M. anguillicaudatus and P. dabryanus, the role of visual signals in mating behavior is presumed to be relatively weak, whereas the role of olfactory signals is believed to be prominent, hence the sex pheromone-receptor system probably plays a key role in species recognition during mating. The selection for the divergence of the sex pheromonereceptor systems of the two species in reinforcement likely have promoted the adaptive evolution of Ma_or114-1a.

\section{Conclusions}

Through trans-species comparative transcriptomic analysis and qRT-PCR assay of three closely related loach species, we found a male-biased expression pattern of the olfactory receptor gene or114-1 shared by the three species. Hence it was inferred that OR114-1 
might be a sex pheromone receptor in the loach species, which was in concordance with the OR114-1 function in zebrafish that was revealed in the previous study. As with zebrafish, M. bipartitus and P. dabryanus both possessed one or114-1; whereas in M. anguillicaudatus, or114-1 has two members: Ma_or114-1a and Ma_or114-1b. Among the two or114-1 members of $M$. anguillicaudatus, the one with sex-differential expression is Ma_or114-1a. These two members have undergone specific changes: Ma_or $114-1 b$ was delayed of the stop codon due to insertions, and the protein sequence length was extended by 8 amino acids; Ma_or114-1a underwent positive selection, and the adaptive amino acid substitution occurred. The ligand binding specificity of the duplicates relative to the ancestral receptor before the duplication probably has changed considering the tremendous sequence change, which implicates the recognition profile of the sex pheromone of the receptors in change. This adaptive evolution might be driven by the co-effect of sexual selection and reinforcement.

Supplementary Materials: The following are available online at https:/ / www.mdpi.com/article/ 10.3390/genes12121845/s1, Additional file 1: Alignment of representative transcripts of or114-1 of the three loach species, Additional file 2: Allele sequences of or114-1 of the three loach species, Additional file 3: Contigs containing or114-1, Figure S1: The relative expression levels of or114 in different male and female individuals of Misgurnus bipartitus, Figure S2: The relative expression levels of or114-1 in different male and female individuals of Paramisgurnus dabryanus, Table S1: Gene differential expression analysis based on RNA-seq data, Table S2: Test result of RELAX, Table S3: Test result of positive selection by aBSREL, Table S4: Test result of positive selection by BUSTED.

Author Contributions: Conceptualization, L.Z.; methodology, L.Z.; software, L.Z.; validation, L.Z. and X.C.; formal analysis, L.Z.; investigation, L.Z.; resources, L.Z., X.C. and W.W.; data curation, L.Z.; writing—original draft preparation, L.Z.; writing—review and editing, L.Z.; visualization, L.Z.; supervision, W.W.; project administration, X.C. and W.W.; funding acquisition, L.Z. and X.C. All authors have read and agreed to the published version of the manuscript.

Funding: This research was funded by the National Natural Science Foundation of China, grant number 31702015; the Fundamental Research Funds for the Central Universities of China (grant numbers 2662018QD052 and 2662020SCPY002); China Agriculture Research System CARS-46.

Institutional Review Board Statement: The study was conducted according to the guidelines of the national legislation of China, and approved by the Ethics Committee of Huazhong Agricultural University (protocol code HZAUFI-2016-006).

Data Availability Statement: Publicly available datasets were analyzed in this study. This data can be found here: [SRA Accession No. SRR14800568-14800573; GenBank Accession No. MZ065164MZ065167].

Conflicts of Interest: The authors declare no conflict of interest. The funders had no role in the design of the study; in the collection, analyses, or interpretation of data; in the writing of the manuscript, or in the decision to publish the results.

\section{References}

1. Smadja, C.; Butlin, R.K. On the scent of speciation: The chemosensory system and its role in premating isolation. Heredity 2009, 102, 77-97. [CrossRef] [PubMed]

2. Seehausen, O.; Terai, Y.; Magalhaes, I.S.; Carleton, K.L.; Mrosso, H.D.; Miyagi, R.; van der Sluijs, I.; Schneider, M.V.; Maan, M.E.; Tachida, H.; et al. Speciation through sensory drive in cichlid fish. Nature 2008, 455, 620-626. [CrossRef] [PubMed]

3. Zhang, B.; Zhang, W.; Nie, R.E.; Li, W.Z.; Segraves, K.A.; Yang, X.K.; Xue, H.J. Comparative transcriptome analysis of chemosensory genes in two sister leaf beetles provides insights into chemosensory speciation. Insect. Biochem. Mol. 2016, 79, 108-118. [CrossRef] [PubMed]

4. Symonds, M.R.E.; Elgar, M.A. The evolution of pheromone diversity. Trends Ecol. Evol. 2008, 23, 220-228. [CrossRef]

5. Poncelet, G.; Shimeld, S.M. The evolutionary origins of the vertebrate olfactory system. Open Biol. 2020, 10, 200330. [CrossRef]

6. Touhara, K.; Vosshall, L.B. Sensing Odorants and Pheromones with Chemosensory Receptors. Annu. Rev. Physiol. 2009, 71, 307-332. [CrossRef]

7. Gomez-Diaz, C.; Benton, R. The joy of sex pheromones. Embo Rep. 2013, 14, 874-883. [CrossRef]

8. Niehuis, O.; Buellesbach, J.; Gibson, J.D.; Pothmann, D.; Hanner, C.; Mutti, N.S.; Judson, A.K.; Gadau, J.; Ruther, J.; Schmitt, T. Behavioural and genetic analyses of Nasonia shed light on the evolution of sex pheromones. Nature 2013, 494, 345-348. [CrossRef]

9. Doyle, W.I.; Meeks, J.P. Excreted Steroids in Vertebrate Social Communication. J. Neurosci. 2018, 38, 3377-3387. [CrossRef] 
10. Bear, D.M.; Lassance, J.M.; Hoekstra, H.E.; Datta, S.R. The Evolving Neural and Genetic Architecture of Vertebrate Olfaction. Curr. Biol. 2016, 26, R1039-R1049. [CrossRef]

11. Haga-Yamanaka, S.; Ma, L.M.; He, J.; Qiu, Q.; Lavis, L.D.; Looger, L.L.; Yu, C.R. Integrated action of pheromone signals in promoting courtship behavior in male mice. Elife 2014, 3, e03025. [CrossRef]

12. Sorensen, P.W.; Stacey, N.E. Brief review of fish pheromones and discussion of their possible uses in the control of non-indigenous teleost fishes. N. Z. J. Mar. Fresh 2004, 38, 399-417. [CrossRef]

13. Appelt, C.W.; Sorensen, P.W. Female goldfish signal spawning readiness by altering when and where they release a urinary pheromone. Anim. Behav. 2007, 74, 1329-1338. [CrossRef]

14. Plenderleith, M.; van Oosterhout, C.; Robinson, R.L.; Turner, G.F. Female preference for conspecific males based on olfactory cues in a Lake Malawi cichlid fish. Biol. Lett. 2005, 1, 411-414. [CrossRef]

15. Shi, P.; Zhang, J. Extraordinary Diversity of Chemosensory Receptor Gene Repertoires Among Vertebrates. In Chemosensory Systems in Mammals, Fishes, and Insects; Meyerhof, W., Korsching, S., Eds.; Springer: Berlin/Heidelberg, Germany, 2009; pp. 57-75.

16. Nei, M.; Niimura, Y.; Nozawa, M. The evolution of animal chemosensory receptor gene repertoires: Roles of chance and necessity. Nat. Rev. Genet. 2008, 9, 951-963. [CrossRef]

17. Churcher, A.M.; Hubbard, P.C.; Marques, J.P.; Canario, A.V.M.; Huertas, M. Deep sequencing of the olfactory epithelium reveals specific chemosensory receptors are expressed at sexual maturity in the European eel Anguilla anguilla. Mol. Ecol. 2015, 24, 822-834. [CrossRef]

18. Yabuki, Y.; Koide, T.; Miyasaka, N.; Wakisaka, N.; Masuda, M.; Ohkura, M.; Nakai, J.; Tsuge, K.; Tsuchiya, S.; Sugimoto, Y.; et al. Olfactory receptor for prostaglandin F-2 $\alpha$ mediates male fish courtship behavior. Nat. Neurosci. 2016, 19, 897-904. [CrossRef]

19. Behrens, M.; Frank, O.; Rawel, H.; Ahuja, G.; Potting, C.; Hofmann, T.; Meyerhof, W.; Korsching, S. ORA1, a Zebrafish Olfactory Receptor Ancestral to All Mammalian V1R Genes, Recognizes 4-Hydroxyphenylacetic Acid, a Putative Reproductive Pheromone. J. Biol. Chem. 2014, 289, 19778-19788. [CrossRef]

20. Cong, X.; Zheng, Q.; Ren, W.; Cheron, J.B.; Fiorucci, S.; Wen, T.; Zhang, C.; Yu, H.; Golebiowski, J.; Yu, Y. Zebrafish olfactory receptors ORAs differentially detect bile acids and bile salts. J. Biol. Chem. 2019, 294, 6762-6771. [CrossRef]

21. Saraiva, L.R.; Ahuja, G.; Ivandic, I.; Syed, A.S.; Marioni, J.C.; Korsching, S.I.; Logan, D.W. Molecular and neuronal homology between the olfactory systems of zebrafish and mouse. Sci. Rep. 2015, 5, 11487. [CrossRef]

22. Wang, Y.; Jiang, H.; Yang, L. Transcriptome Analysis of Zebrafish Olfactory Epithelium Reveal Sexual Differences in Odorant Detection. Genes 2020, 11, 592. [CrossRef] [PubMed]

23. Kolmakov, N.N.; Kube, M.; Reinhardt, R.; Canario, A.V. Analysis of the goldfish Carassius auratus olfactory epithelium transcriptome reveals the presence of numerous non-olfactory GPCR and putative receptors for progestin pheromones. BMC Genom. 2008, 9, 429. [CrossRef] [PubMed]

24. Fatsini, E.; Bautista, R.; Manchado, M.; Duncan, N.J. Transcriptomic profiles of the upper olfactory rosette in cultured and wild Senegalese sole (Solea senegalensis) males. Comp. Biochem. Physiol. Part D Genom. Proteom. 2016, 20, 125-135. [CrossRef] [PubMed]

25. Palstra, A.P.; Fukaya, K.; Chiba, H.; Dirks, R.P.; Planas, J.V.; Ueda, H. The Olfactory Transcriptome and Progression of Sexual Maturation in Homing Chum Salmon Oncorhynchus keta. PLoS ONE 2015, 10, e0137404.

26. Zhu, G.; Wang, L.; Tang, W.; Liu, D.; Yang, J. De novo transcriptomes of olfactory epithelium reveal the genes and pathways for spawning migration in japanese grenadier anchovy (Coilia nasus). PLoS ONE 2014, 9, e103832.

27. Liu, H.; Chen, C.; Lv, M.; Liu, N.; Hu, Y.; Zhang, H.; Enbody, E.D.; Gao, Z.; Andersson, L.; Wang, W. A chromosome-level assembly of blunt snout bream (Megalobrama amblycephala) reveals an expansion of olfactory receptor genes in freshwater fish. Mol. Biol. Evol. 2021, 38, 4238-4251. [CrossRef]

28. Honda, H. Female sex pheromone of the loach, Misgurnus anguillicaudatus, involved in courtship behavior. Bull. Jpn. Soc. Sci. Fish 1980, 46, 1223-1225. [CrossRef]

29. Kitamura, S.; Ogata, H.; Takashima, F. Activities of F-type prostaglandins as releaser sex pheromones in cobitide loach, Msgurnus anguillicaudatus. Comp. Biochem. Physiol. Part A Physiol. 1994, 107, 161-169. [CrossRef]

30. Chen, J. A study on the classification of the subfamily cobitinae of china. Trans. Chin. Ichthyol. Soc. 1981, 1, 21-32.

31. Fujimoto, T.; Nishimura, T.; Goto-Kazeto, R.; Kawakami, Y.; Yamaha, E.; Arai, K. Sexual dimorphism of gonadal structure and gene expression in germ cell-deficient loach, a teleost fish. Proc. Natl. Acad. Sci. USA 2010, 107, 17211-17216. [CrossRef]

32. Yu, Y.Y.; Abbas, K.; Wang, W.M.; Zhou, X.Y. Geographical distribution of ploidy level variation of loach Misgurnus anguillicaudatus in China. Pak. J. Agr. Sci. 2014, 51, 273-281.

33. Zhong, J.; Yi, S.; Ma, L.; Wang, W. Evolution and phylogeography analysis of diploid and polyploid Misgurnus anguillicaudatus populations across China. Proc. Biol. Sci. 2019, 286, 20190076. [CrossRef]

34. Grabherr, M.G.; Haas, B.J.; Yassour, M.; Levin, J.Z.; Thompson, D.A.; Amit, I.; Adiconis, X.; Fan, L.; Raychowdhury, R.; Zeng, Q.; et al. Full-length transcriptome assembly from RNA-Seq data without a reference genome. Nat. Biotechnol. 2011, $29,644-652$. [CrossRef]

35. Pertea, G.; Huang, X.; Liang, F.; Antonescu, V.; Sultana, R.; Karamycheva, S.; Lee, Y.; White, J.; Cheung, F.; Parvizi, B.; et al. TIGR Gene Indices clustering tools (TGICL): A software system for fast clustering of large EST datasets. Bioinformatics 2003, 19, 651-652. [CrossRef]

36. Audic, S.; Claverie, J.M. The significance of digital gene expression profiles. Genome Res. 1997, 7, 986-995. [CrossRef] 
37. Kumar, S.; Stecher, G.; Tamura, K. MEGA7: Molecular Evolutionary Genetics Analysis Version 7.0 for Bigger Datasets. Mol. Biol. Evol. 2016, 33, 1870-1874. [CrossRef]

38. Ronquist, F.; Huelsenbeck, J.P. MrBayes 3: Bayesian phylogenetic inference under mixed models. Bioinformatics 2003, 19, 1572-1574. [CrossRef]

39. Livak, K.J.; Schmittgen, T.D. Analysis of relative gene expression data using real-time quantitative PCR and the 2(-Delta Delta C(T)) Method. Methods 2001, 25, 402-408. [CrossRef]

40. Yang, Z.H. PAML 4: Phylogenetic analysis by maximum likelihood. Mol. Biol. Evol. 2007, 24, 1586-1591. [CrossRef]

41. Wertheim, J.O.; Murrell, B.; Smith, M.D.; Pond, S.L.K.; Scheffler, K. RELAX: Detecting Relaxed Selection in a Phylogenetic Framework. Mol. Biol. Evol. 2015, 32, 820-832. [CrossRef]

42. Smith, M.D.; Wertheim, J.O.; Weaver, S.; Murrell, B.; Scheffler, K.; Kosakovsky Pond, S.L. Less is more: An adaptive branch-site random effects model for efficient detection of episodic diversifying selection. Mol. Biol. Evol. 2015, 32, 1342-1353. [CrossRef] [PubMed]

43. Murrell, B.; Weaver, S.; Smith, M.D.; Wertheim, J.O.; Murrell, S.; Aylward, A.; Eren, K.; Pollner, T.; Martin, D.P.; Smith, D.M.; et al. Gene-wide identification of episodic selection. Mol. Biol. Evol. 2015, 32, 1365-1371. [CrossRef] [PubMed]

44. Hofmann, K. TMBASE_A database of membrane spanning protein segments. Biol Chem. 1993, 374, 1-3.

45. Alioto, T.S.; Ngai, J. The odorant receptor repertoire of teleost fish. BMC Genom. 2005, 6, 173. [CrossRef]

46. Bushdid, C.; de March, C.A.; Fiorucci, S.; Matsunami, H.; Golebiowski, J. Agonists of G-Protein-Coupled Odorant Receptors Are Predicted from Chemical Features. J. Phys. Chem. Lett. 2018, 9, 2235-2240. [CrossRef]

47. Zhang, J.Z. Evolution by gene duplication: An update. Trends Ecol. Evol. 2003, 18, 292-298. [CrossRef]

48. Ortiz-Barrientos, D.; Counterman, B.A.; Noor, M.A. The genetics of speciation by reinforcement. PLoS Biol. 2004,2 , e416. [CrossRef]

49. Spencer, H.G. A further perspective on speciation by reinforcement. Theor. Biol. Forum. 2020, 113, 63-66.

50. Butlin, R.K.; Smadja, C.M. Coupling, Reinforcement, and Speciation. Am. Nat. 2018, 191, 155-172. [CrossRef]

51. Servedio, M.R.; Noor, M.A.F. The Role of Reinforcement in Speciation: Theory and Data. Annu. Rev. Ecol. Evol. Syst. 2003, 34, 339-364. [CrossRef]

52. Matute, D.R.; Ortiz-Barrientos, D. Speciation: The strength of natural selection driving reinforcement. Curr. Biol. 2014, 24, R955-R957. [CrossRef] 